\title{
The Blend of Toughness and Delicacy: The Interpretation of Tigress's Tragedy Fate Based on the Modern Vision
}

\author{
Chunyu Dong \\ School of Liberal Arts, Anhui University, Hefei, China \\ Email: 1301074050@qq.com
}

How to cite this paper: Dong, C.Y. (2020) The Blend of Toughness and Delicacy: The Interpretation of Tigress's Tragedy Fate Based on the Modern Vision. Open Access Library Journal, 7: e6917.

https://doi.org/10.4236/oalib.1106917

Received: October 21, 2020

Accepted: November 17, 2020

Published: November 20, 2020

Copyright $\odot 2020$ by author(s) and Open Access Library Inc.

This work is licensed under the Creative Commons Attribution International License (CC BY 4.0).

http://creativecommons.org/licenses/by/4.0/

\begin{abstract}
Tigress is the most important female character in Laoshe's novella Camel Xiangzi. Readers used to think Tigress was insidious and cunning, so far as to exaggerate the influence of Tigress on Xiangzi's fate tragedy. Of course, Tigress has some human weaknesses, but this should not be the reason why readers criticize her wantonly. Ugly appearance and vulgar manners won't obscure Tigress's delicate and sincere heart.
\end{abstract}

\section{Subject Areas}

Literature

Keywords

Camel Xiangzi, Tigress, Fate Tragedy

\section{1. 引言}

《骆驼祥子》是一部老舍最满意的作品, 它打响了老舍成为 “职业写家 的第一炮(老舍《我怎样写<骆驼祥子>》)” [1], 堪称是我国现代文学的经典。 《骆驼祥子》主要讲述的是旧北京人力车夫祥子的辛酸故事。作品以祥子买 车 “三起三落” 为情节发展的中心线索, 淋漓尽致地表现了旧社会底层劳动 人民的苦难生活, 展现了祥子一步步走向堕落的悲惨一生。这部作品塑造了 以祥子为代表的众多经典人物形象, 而人和车厂的女儿、祥子的妻子虎妞就 是其中的典型代表。

由于时代等作品本身难以避免的局限, 读者在阅读过程中常常会对虎妞 形成一种片面的误读。一般普遍认为, 在作品中, 虎妞用 “怀孕” 欺骗祥子 与其结婚, 以及婚后对祥子的性压椥等是祥子人生悲剧的重要缘由, 以致后 
来许多读者在谈及虎妞时往往总是看到她虚伪狡诈的一面, 说她粗鲁跋扈、 好色淫荡、贪吃纵欲、丑唒庸俗等等, 甚至过分夸大虎妞对祥子人生悲剧造 成的影响。在笔者看来, 虎妞确实是存在一些贪婪、自私等人性弱点, 但她 却并不是一个应该被大肆批判的人。相貌丑婳、举止粗俗并不应该成为全盘 否定虎妞这一人物形象的理由, 为此我们应该跳出时代的局限, 用一种更加 现代的眼光来解读虎妞这一人物形象。

\section{2. 彪悍与细淢的交融}

虎妞自幼丧母，由父亲刘四爷抚养、在一个聚集了粗鄙的下层男人的人 和车厂长大，父亲刘四爷 “当过库兵，设过奢场，买卖过人口，放过阎王账， 干这些营生所应有的资格与本领一一力气、心路、自豪等等一一刘四爷都有”, 车厂里的车夫有着 “可以原谅而不可效法的恶习……生活的毒疮只能借着烟 酒妇人的毒药麻木一会, 以毒攻毒, 毒气有朝一日必会归了心”, 在这种环 境下长大, 耳濡目染间, 虎妞举止粗野如男人, “干什么都和男人一样, 连 骂人也有男人的爽快” ，这一点可以说并不是她的错。尽管她言辞粗鄙却也 并不是一个大条无脑的女子，她也会温柔会细心，当祥子身处困境时她貌似 责骂的凶言凶语中掩藏的其实是真诚的关心，当她敏感地察觉出祥子情绪低 落时, 她也会及时刹住即将脱口而出的不合适的话语。祥子从山里牵着骆驼 死里逃生跑回人和车厂, 虎妞会热情的招呼他 “要是没吃了的话, 一起吃吧”, 难怪祥子会一直拿人和车厂作家, 受到虎妞热情的招待, 心里也会忽然感受 到一点说不出来的亲热。祥子为了麻痹被大兵抢车的痛苦, 不顾命地拉车赚 钱, 虎妞不像刘四爷和其他车夫那样幸灾乐祸的看热闹, 而是嘱咐他好几回 “你这家伙要是这么干，吐了血可是你自己的事……买车也得悠停着来，当 你是铁做的哪！你应当好好的歇三天”, 对祥子, 虎妞也是 “一百一的客气, 爱护”。由此我们不难看出，虎妞实则是属于那种 “刀子口，豆腐心” 的女 人, 看似粗野如男人, 实则内心丰富, 外表举止的粗䊁并不会掩盖她内心的 细淢与真诚。

粗粘与细淢看似矛盾而又统一地交融在了虎妞一人的身上，使得虎妞在 处理个人情感上也是刚爱敢恨, 绝不拖泥带水。由于父亲的私心和当时男人 们的择偶观念，虎妞三十多岁仍没有嫁出去。但虎妞并没有过多地怨天尤人， 而是积极主动地去追求自己的爱情。作为一个女人，她尊敬祥子、爱慕祥子, 在日常生活中毫不掩饰地表达自己对祥子的喜爱, 并丝毫不忌讳外人的眼光。 有读者认为虎妞十分阴险狡计，欺骗祥子与自己结婚，以致毁了祥子的人生。 我国传统观念往往认为女子应该是含蓄内敛的, 而女子主动追求爱情婚姻幸 福则是不符合 “三从四德” 的规矩的。但在我国古代文学作品中我们却常常 注意到, 许多女子都会大胆追求自己的幸福, 比如在《诗经》中就有 “风雨 如晦, 鸡鸣不已, 既见君子, 云胡不喜”、“子惠思我, 褰裳涉溱。子不思 我, 岂无他人? 狂童之狂也且? ” [2]等诗句, 《天仙配》、《梁祝》、《西 厢记》、《白蛇传》等更是我们所熟悉的 “女追男” 的经典故事代表, 在这 些故事中，女主人公热情地追求自己的心意的男子，甚至不惜采取一些小阴 
谋、小花招, 虎妞与这些女子的计谋比起来, 可以说是 “小巫见大巫” 了。 但是为什么历代读者对以上这些女子赞赏有加, 却对虎妞大肆批判呢, 笔者 认为, 还归根到底是因为虎妞与以上那些女子相比, 她 “老、丑、不美”, 固然爱美之心人皆有之, 但从外表的批判上升到道德的批判就难免有失偏颇 了。

\section{3. 事业上的 “女强人”与生活上的“贤内助”}

在小说中, 虎妞虽然样貌丑陃, 其实上既聪明又能干。虎妞一出场, 作 者就开门见山地指出 “帮助父亲办事是把好手……刘四爷打外, 虎妞打内, 父女把人和车厂治理的铁桶一般。人和车厂成了养车界的权威, 刘家父女的 办法常常在车夫与车主口上, 如读书人的引经据典。” 短短几句话, 就描写 出了虎妞的精明能干的特质, 她也为此得到了包括祥子在内一众车夫的敬重, 车夫们在虎妞面前不敢要滑头。且不论小说将虎妞的生活背景设置在一个男 尊女卑的旧社会, 就算在我们较为开放公正的当下社会, 虎妞这类女子在治 理事业上也绝对算得上是一个 “女强人”。

虎妞除了对外治理事业上是一个 “女强人”, 她在处理家庭生活上也堪 称是一个 “贤内助”。在婚后的 “家政” 上, 虎妞更是表现她的贤惠来, 她 把新房装饰的喜庆温暖, 做饭, 收拾屋子, “一举一动都像个多年的媳妇, 麻利, 老道”, 让孤苦一人的祥子 “觉得自己是有了家”。在笔者看来, 虎 妞虽然是个三十多岁的老姑娘倒也 “御夫有道” 。我们现代有一句话说 “要 想留住一个男人的心, 先留住他的胃”, 虎妞看来也是深谙此道, 婚后经常 给祥子改善伙食, 时不时还会买些零嘴, 祥子自己也不得不承认 “婚后的饭 食的确是比平日的可口, 热火”。婚前虎妞掌管车厂大权, 雷厉风行, 说一 不二, 稍稍不顺心就开骂, 婚后虎妞关于家庭事务倒也会与丈夫商议, 必要 时甚至会做出让步, 虎妞晓得 “祥子并不是个蚌驴……她颇得用点儿心思才 能拢得住这个急了也会地蹶子的大人, 或是大东西。她不能逼太紧了, 找这 么个大东西不是件很容易的事。她得松一把, 紧一把, 叫他老逃不出她的手 心儿。”这也难怪祥子婚后对虎妞又憎又恶又不敢冲撞她, 就连虎妞死后祥 子也想 “虎妞虽然厉害, 但没有了她怎能成个家呢? ”

\section{4. 传统思想与个人欲望的冲突}

关于结婚, 起初祥子的理想是 “必娶一个一清二白的姑娘……必定到乡 下娶个年轻力壮, 吃得苦, 能洗能做的姑娘”, 可见祥子对婚姻的态度还是 相当传统保守的。在小说中, 祥子受到虎妞直接主动地暗示、诱惑, 与虎妞 发生了一夜情, 但是事后却发现虎妞早已不是处女, 而在这一夜之前自己却 是一个对男女关系尚且懵懂无知的处男。尽管是祥子自己没有经受住诱惑, 自愿上了虎妞的床, 祥子对虎妞的反感却也加剧了, 把这看作是人生的污点, 感觉 “不但身上好像沾了点什么, 心中也好像多了一个黑点, 永远不能洗去”。 从此以后, 祥子对虎妞的态度也是日益恶化, 对她的称呼也从 “虎姑娘” 到 直呼其名 “虎妞”, 再到后来 “吸人精血的东西”、“女怪”！但实际上, 
虎妞并没有所谓的那么淫荡放纵, 小说中也明白地指出 “并没有听说过她有 什么不规矩的地方; 虽然她对大家很随便爽快, 可是大家并没有在背地讲论 过她; 即使车夫中有说她坏话的, 也是说她厉害, 没有别的。” 在车厂那种 下等男性车夫聚集、人多嘴杂的地方, 虎妞能不被车夫背后说闲话, 从这一 点也能看出虎妞也是较为清白的。婚后虎妞对祥子的 “性压榕” 更是让许多 读者将其视为洪水猛兽, 但如果我们从另一个角度来理解, 虎妞那时已经将 近四十岁，当了三十多年的老姑娘难免在性爱方面会有所渴望，用她自己的 话来说就是要 “充分享受新婚的快乐......要捞回这点欠缺”。

另外也有一些读者指出, 虎妞在婚后贪图安逸享乐, 生活挥霍无度, 以 致生孩子难产而无钱救治, 直接导致了自己的死亡。但换另一角度思考, 虎 妞并不是穷人家的姑娘，自小便没有勤俭节约、艰苦奋斗的习惯，以刘家在 京城的财力, 她大可以像其他阔太太、大小姐一样整日穿金戴银、吃喝玩乐, 但是虎妞除了好吃些零嘴之外, 她平日里也是不施粉黛, 衣着也是相当朴素, 难怪祥子突然某天看到虎妞化妆打扮起来, 会觉得她 “带出些媚气”，“心 里忽然感到点不好意思”。婚后虎妞与刘四爷彻底闹秎, 净身出户嫁给祥子, 虽说应该 “嫁鸡随鸡，嫁狗随狗”，安于清贫，节俭自律，但人打小的形成 的习惯也不是说改就能改的，更何况虎妞对祥子已经一再退步忍让，还将自 己的积蓄拿出来让祥子买车。在十九章, 虎妞难产, 出于对命运的抗争和对 生的希望, 虎妞彻底悔过, 她拿出最后的七八块钱来要祥子去请神婆 “好祥 子, 快快去吧！花钱不要紧！等我好了, 我乘乘跟你过日子！快去吧！”一 个心高气傲的女人能放下曾经拥有的一切, 为她所喜爱的男人做出改变, 还 有什么理由再责骂她呢。

\section{5. 小说中的其他女性形象}

在小说中除了虎妞这一主要女性形象之外，也有其他的女性，她们的出 现一方面是为了展示祥子的一步步墮落, 另一方面也起到了侧面祄托虎妞的 作用。祥子在京郊的农村长大，他对于女人的观念还是封建保守的，就连买 了新车做买卖, 他也认为 “头一个买卖必须拉个穿得体面的人, 绝对不能是 个女的”。小说中祥子一共欣赏过三个女子, 第一个女子是曹太太, 曹宅是 祥子所混过的宅门里, 最可爱的, 曹先生和曹太太都和和气，小说中曹太太 温柔体贴， “也规规矩矩的得人心”; 第二个女子是祥子的邻居兼朋友小福 子，小福子长得不难看，有着一口白牙，做过军官的小，当过妓女，按理说 小福子并不属于祥子娶媳妇 “规规矩矩、一清二白” 的标准, 但祥子还是很 喜欢她, “她美, 她年轻, 她要强, 她勤俭, 如果想再娶, 她是个理想的人”, 可见祥子还是喜欢像曹太太、小福子那样面容姣好、三从四德的女人，甚至 在三从四德面前, 所谓的贞洁也可以先向后一放, 就连凶恶的虎妞极少时低 柔地喊了一声 “祥子” ，祥子也会 “怒气打散了好些，那声 “祥子” 还在心 中微微的响着, 换起些无可否认的, 欲断难断的, 情分, 说话还是低声的, 但是温和了些” ，试想虎妞要是放下她的野蛮与强制，稍稍温柔些，她与祥 子的夫妻生活会更舒畅些; 第三个女人是曹家女佣高妈, 书中没有对高妈过 
多地外貌描写, 想必她也只是一个样貌平平的中年妇女, 高妈中年守寡还放 高利贷, 但她 “干净, 爽快做事麻利又仔细。在别处有人嫌她太张道, 注意 多, 时常有些神眉鬼道儿……比一般男子还有心路与能力, 她的话是抄跟儿 来的”, 读到这段描述笔者可能第一个便会联想到, 这不恰恰是虎妞的性格 和作风吗, 但祥子对高妈是相当的敬佩, 很愿意听她说话, 每逢遇到他, 祥 子会俊俊乎乎的一笑, 这与对虎妞的态度是截然相反的。说到底, 还是因为 虎妞凶、丑。第四章虎妞一出场, 老舍就描写道她 “一脸虎相……长得虎头 虎脑”。我们一般用 “虎头虎脑” 来形容小男孩, 作者冷不丁将 “虎头虎脑” 用在一个三十多岁的老姑娘身上, 不禁叫人亚然失笑。曹宅的高妈曾描述虎 妞 “像个大黑塔! 怪吓人的! ”, 可见虎妞是真的相貌凶恶丑陃, 甚至化了 妆祥子都觉得 “脸上大概又擦了粉, 被灯照的显出点儿灰绿色, 像枯黑了的 树叶上挂着层霜” 。对比虎妞与曹太太、小福子、高妈这三个女性, 我们可 以得出这样的结论, 祥子不爱虎妞, 其实只是因为虎妞的容貌无法取悦他, 虎妞的才能压制了他, 虎妞的强制威胁到了他。

\section{6. 结语}

老舍被称为是 “人民艺术家” ，他的作品贴近市井气息，敢于为底层的 劳动人民说话, 表达底层人民的真善美、无奈和不平。但一个有意思的现象 是, 在老舍的作品中, 却少有女性形象的出现, 正面的女性形象更是少之又 少。少数老舍抱有同情心创作的女性的形象则是像《骆驼祥子》里的小福子、 《月牙儿》里的韩月荣等这样的底层妇女。在这一点上, 对比同时代的巴金、 曹禺、沈从文等作家, 老舍作品确实是有些花容失色。搜集老舍的资料可以 知道, 老舍两岁时, 身为清皇城护军的父亲在八国联军战争中阵亡, 此后, 老舍一家人的生活仅靠母亲给人家缝洗衣服和在小学当佣工来维持, 我们想 必也可以推测, 老舍的母亲在他的人生道路上起到了非常重要作用, 但是老 舍作品中却少有传统坚韧包容的伟大妇女形象, 这是一个很值得进一步研究 的问题, 或许能为解读虎妞这一人物形象提供一个新的视角。

援引《〈骆驼祥子〉：民间视角下的启蒙悲剧》中的一段话： “在现实 生活的层面上, 虎妞与祥子在一起的时候, 总是虎妞比祥子更可爱, 而且真 心, 祥子反倒显得虚伪冷酷。虎妞虽然对别人坏, 但对祥子是真心实意的, 而祥子却一边在虎妞身上讨便宜, 一边又把责任推得干干净净。” [3]用我们 现代的眼光来看, 祥子对待工作是一把好手, 但对待感情他是一个不折不扣 的大男子主义者, 将自己的理想与想法一厢情愿地施加到女性身上。虎妞将 自己的幸福与希望统统托付给了祥子, 却换不回充满幸福与希望的结局, 读 了那么多遍的《骆驼祥子》, 总是会为虎妞这个不讨喜欢的老女人而心酸落 泪。

\section{Conflicts of Interest}

The author declares no conflicts of interest regarding the publication of this paper. 


\section{References}

[1] 老舍. 我怎样写《骆驼祥子》[J]. 中华活页文选(初三), 2009(6): 46-48.

[2] 余冠英注. 诗经选 [M]. 第 2 版. 北京: 人民文学出版社, 1979.

[3] 陈思和. 《骆驼祥子》: 民间视角下的启蒙悲剧[J]. 陕西师范大学学报(哲学社会 科学版), 2004, 33(3): 5-16.

\section{Appendix (Abstract and Keywords in Chinese)}

\section{彪悍与细淢的交融: 基于现代视野下的虎妞命运悲剧解读}

摘要: 虎妞是老舍中篇小说《骆驼祥子》中最重要的女性形象。以往的 读者往往会认为虎妞阴险狡诈, 甚至过分夸大虎妞对祥子命运悲剧性的影响。 其实不然, 虎妞固然存在一些人性弱点, 但这不应成为读者们大作批判她的 理由, 外表举止的彪悍粗俗并不能掩盖她内心的细淢与真诚。

关键字: 《骆驼祥子》, 虎妞, 爱情悲剧 